\title{
NOMBRES COMUNES DE LAS AVES DE COSTA RICA: SIGNIFICADO Y ORIGEN
}

\author{
Luis Sandoval
}

\begin{abstract}
RESUMEN
Este trabajo describe una compilación de los nombres comunes para las aves de Costa Rica. Además se incluye una explicación de su significado, con los lugares de procedencia y uso de estos nombres. La presente lista permitirá una comunicación más fluida entre ornitólogos y personas comunes, con fines de conservación.

Palabras clave: aves de Costa Rica, nombres comunes, ornitología.
\end{abstract}

\begin{abstract}
This paper is a compilation of the common name of the Costa Rican birds. Also are including a name explanation as the origin and uses places. Update list would permit a better communication among ornithologist and common people in conservation works.

Key words: Costa Rican birds, common names, ornithology.
\end{abstract}

\section{Introducción}

\subsection{Antecedentes de la nomenclatura}

Actualmente, todas las especies de organismos conocidos poseen un nombre único que facilita la comunicación entre científicos del mundo, el cual está basado en un sistema binomial propuesto por Linneus hace cerca de 250 años. Dada su facilidad tanto taxonómica como de comunicación, se sigue utilizando actualmente, pese a las críticas que se han generado recientemente (Härlin 2003: 138, Polaszek y Wilson 2005: 421). Este nombre, por lo general, es poco variable; sin embargo, existen al menos tres causas que generan estas permutaciones: un aumento en el conocimiento de los organismos, para que exista concordancia entre el binomio o por prioridad en el nombramiento de la especie (Banks et al. 2002: 987). Tales cambios son regulados por el Código Internacional de Nomenclatura Zoológica (ICZN por sus siglas en inglés).

Luis Sandoval. Bachiller en Biología, Universidad de Costa Rica.

Correo electrónico: biosandoval@hotmail.com

Recepción: 4-5-2006

Aprobación: 26-6-2006 


\subsection{Uso de nombres comunes en Costa Rica}

En Costa Rica, los nombres comunes varían mucho entre lugares (Quesada-Monge y Fernandez-Vega 2005: 2), debido a que no todos los pueblos utilizan las mismas características para designar una especie, además de que el habla es un elemento muy plástico, que se adapta y se modela dependiendo de las necesidades de los hablantes y lo que se quiera transmitir.

En vista de lo anterior, Fournier y García (1998) realizaron un esfuerzo por compilar los nombres comunes de las plantas de Costa Rica, lo que constituyó el primer intento serio por plasmar en papel y dar a conocer esta parte de la cultura a la ciencia y el pueblo. En cuanto a los animales, poco se ha hecho en este sentido, pues lo que se ha acostumbrado en las guías o libros sobre estas especies costarricenses es utilizar como nombres comunes los nombres en inglés (Savage 2002) o traducciones de estos como de uso primario (Stiles y Skutch 1989), aunque se hagan referencia a algunos nombres comunes. Una excepción a lo anterior es Mora (2000), quien en su libro compila nombres comunes para mamíferos silvestres de Costa Rica y propone otros más acordes con nuestra cultura.

\subsection{Objetivo de la investigación}

La utilización de nombres en español para designar especies animales o vegetales siempre ha suscitado una amplia discusión, especialmente cuando se quieren proponer nombres estandarizados (Vuilleumier 1999: 71). En Costa Rica, se cuenta con al menos 857 especies de aves hasta la fecha (Barrantes et al. 2002: 13), lo que ha permitido que el pueblo tenga contacto con ellas de forma constante. Esta interacción ha generado que se nombre principalmente aquellas aves que representan un interés para él o que son más comunes (Mones y Kuhl de Mones 1991: 250). Debido a la diversidad de nombres comunes que se utilizan en el país para denominar las aves, nuestro trabajo constituye el primer esfuerzo de compilación de este tema, el cual facilitará la comunicación entre científicos y personas comunes en el campo de la ornitología en el país. La meta de este trabajo es dar a conocer la diversidad de los nombres comunes para algunas aves de Costa Rica, con su significado y lugar de procedencia o utilización.

\section{Metodología}

\subsection{Recolección de datos}

La colecta de los nombres comunes se realizó durante los últimos cinco años de tres diferentes formas. La primera, por consulta explícita o por hablar con los pobladores de los lugares durante giras de campo. La segunda, por preguntar a pajareros (personas que cazan aves por canto o plumaje para mantenerlas como mascotas). La tercera, por charlas con colegas de la Universidad de Costa Rica, quienes facilitaron los nombres que ellos han escuchado en otros sitios del país durante sus giras.

\subsection{Origen y significado de los nombres}

Durante la investigación, se trató de averiguar el significado del nombre y se decidió establecer una clasificación con base en cuatro categorías: 
1. Físico: cuando describe algún rasgo morfológico del ave.

2. Comportamiento: cuando se describe algún comportamiento del ave.

3. Onomatopéyico: cuando se asocia con alguna vocalización o sonido hecho por el ave.

4. Geográfico: cuando esta asociado a un hábitat o lugar de origen.

También se anotó el lugar de procedencia o uso del nombre. Esta clasificación varía desde pueblos hasta el país como un todo. Además, se incluye como sitio de origen a los pajareros, los cuales no se circunscriben a un lugar geográfico específico, y que por comunicación entre ellos han expandido nombres entre zonas.

\section{Análisis de datos}

A la fecha, se han recopilado un total de 309 nombres comunes de 238 especies de 57 familias, a lo largo de todo el país (Cuadro 1). La familia Thraupidae es la que presentó más nombres comunes con 31 y más especies con nombres comunes con 22 (Cuadro 1). En total, 19 familias solo presentaron una especie con un nombre común (Cuadro 1). Sporophila americana fue la especie que presentó más nombres comunes, con cuatro. El 96 por ciento de las especies presentaron solo uno o dos nombres comunes (Cuadro 1). El nombre de Pecho Amarillo es asignado a un total de siete especies, con lo que se convierte en el nombre común más utilizado. Los pajareros aportan un total de 62 nombres comunes a 58 especies (Cuadro 1).

Cuadro 1. Listado de especies de aves según orden taxonómico y nombres comunes asignados según origen y lugar

\begin{tabular}{llll}
\hline Familia y especie & Nombre común & Lugar & Origen \\
\hline Tinamidae & & & Comportamiento \\
\hline Tinamus major & Gallina de monte & Tortuguero & Onomatopéyico \\
& Yerre & Tortuguero & Comportamiento \\
Nothocercus bonapartei & Gallina de monte & Valle Central & Físico \\
Crypturellus soui & Gongolona & Valle Central & Comportamiento \\
Crypturellus cinnamomeus & Gallina de monte & Atlántico & Comportamiento \\
Crypturellus boucardi & Gallina de monte & Guanacaste & Comportamiento \\
\hline Podicipedidae & Gallina de monte & Atlántico & Comportamiento \\
\hline Tachybaptus dominicus & & & Falle Central \\
\hline Sulidae & Zambullidores & & \\
\hline Sula leucogaster & & Bahía Ballena & \\
\hline Pelecanidae & Monja & Guanacaste & Físico \\
\hline Pelecanus occidentalis & Buchón & Costa Rica & \\
\hline
\end{tabular}




\begin{tabular}{|c|c|c|c|}
\hline \multicolumn{4}{|l|}{ Phalacrocoracidae } \\
\hline Phalacrocorax brasilianus & Pato chancho & Guanacaste & Onomatopéyico \\
\hline \multicolumn{4}{|l|}{ Anhingidae } \\
\hline Anhinga anhinga & Pato aguja & Guanacaste, Sarapiquí & Físico \\
\hline \multicolumn{4}{|l|}{ Fregatidae } \\
\hline Fregata magnificens & Tijereta & $\begin{array}{l}\text { Guanacaste, Puntarenas, } \\
\text { Golfito }\end{array}$ & Físico \\
\hline \multicolumn{4}{|l|}{ Ardeidae } \\
\hline Tigrisoma mexicanum & Garzón & Guanacaste & Físico \\
\hline Egretta caerulea & Garza negra & Sierpe & Físico \\
\hline \multirow[t]{2}{*}{ Bubulcus ibis } & Garcilla bueyera & Valle Central & Comportamiento \\
\hline & Garcilla ganadera & Valle Central & Comportamiento \\
\hline Butorides virescens & Martín peña & Tortuguero & \\
\hline Cochlearius cochlearius & Chocuaco & Guanacaste & Onomatopéyico \\
\hline \multicolumn{4}{|l|}{ Threskiornitidae } \\
\hline \multirow[t]{2}{*}{ Platalea ajaja } & Espátula rosada & Guanacaste & Físico \\
\hline & Garza morena & Guanacaste & Físico \\
\hline \multicolumn{4}{|l|}{ Ciconiidae } \\
\hline Jabiru mycteria & Galán sin ventura & Guanacaste & \\
\hline \multicolumn{4}{|l|}{ Cathartidae } \\
\hline \multirow[t]{2}{*}{ Coragyps atratus } & Zoncho & Valle Central & \\
\hline & Zopilote & Valle Central & \\
\hline \multirow[t]{2}{*}{ Cathartes aura } & Zonchite & Valle Central & \\
\hline & Zopilote & Valle Central & \\
\hline Sarcoramphus papa & Rey de zopilote & Zona Sur & Físico \\
\hline \multicolumn{4}{|l|}{ Anatidae } \\
\hline \multirow[t]{2}{*}{ Dendrocygna autumnalis } & Pijije & Guanacaste & Onomatopéyico \\
\hline & Piche & Guanacaste & Onomatopéyico \\
\hline Dendrocygna bicolor & Piche canelo & Guanacaste & Físico \\
\hline Cairina moschata & Pato real & Guanacaste & Físico \\
\hline \multicolumn{4}{|l|}{ Pandionidae } \\
\hline Pandion haliaetus & Águila pescadora & Cerro de la Muerte & Comportamiento \\
\hline \multicolumn{4}{|l|}{ Accipitridae } \\
\hline Elanoides forficatus & Gavilán tijerilla & Valle Central & Físico \\
\hline \multirow[t]{2}{*}{ Elanus leucurus } & Gavilán bailarín & Valle Central & Comportamiento \\
\hline & Gavilancillo blanco & Valle Central & Físico \\
\hline Geranospiza caerulescens & Caracorelo & Guanacaste & Comportamiento \\
\hline Buteogallus urubitinga & Cangrejero & Tortuguero & Comportamiento \\
\hline Buteo magnirostris & Pata podrida & Osa & Comportamiento \\
\hline Buteo platypterus & Gavilán pollero & Valle Central & Comportamiento \\
\hline \multicolumn{4}{|l|}{ Falconidae } \\
\hline Ibycter americanus & Cacao & Sarapiquí & Onomatopéyico \\
\hline \multirow[t]{2}{*}{ Caracara cheriway } & Caracara & Guanacaste & Onomatopéyico \\
\hline & Kerke & Las Juntas Abangares & Onomatopéyico \\
\hline Milvago chimachima & Gavilán Blanco & San Vito & Físico \\
\hline
\end{tabular}




\begin{tabular}{|c|c|c|c|}
\hline Herpetotheres cachinnans & Guaco & Costa Rica & Onomatopéyico \\
\hline \multicolumn{4}{|l|}{ Cracidae } \\
\hline Ortalis vetula & Chachalaca & Pajareros & Comportamiento \\
\hline Ortalis cinereiceps & Chachalaca & Pajareros & Comportamiento \\
\hline Penelope purpurascens & Pava granadera & Pajareros & Físico \\
\hline Chamaepetes unicolor & Pajuila & Pajareros & Físico \\
\hline Crax rubra & Pavón & Pajareros & Físico \\
\hline \multicolumn{4}{|l|}{ Odontophoridae } \\
\hline Colinus leucopogon & Codorniz de monte & Valle Central & Comportamiento \\
\hline Dendrortyx leucophrys & Chirrascua & Valle Central & Onomatopéyico \\
\hline Odontophorus gujanensis & Julián piojo & Patarra, San José & Onomatopéyico \\
\hline Odontophorus leucolaemus & Guevos de chancho & Pajareros & Onomatopéyico \\
\hline Odontophorus guttatus & Corcovado & Zona sur & Onomatopéyico \\
\hline \multicolumn{4}{|l|}{ Rallidae } \\
\hline \multirow[t]{2}{*}{ Laterallus albigularis } & Freidora & Zona sur & Onomatopéyico \\
\hline & Huevo Frito & Atlántico & Onomatopéyico \\
\hline \multirow{2}{*}{ Aramides cajanea } & Chirincoca & San Vito & Onomatopéyico \\
\hline & Ponpone & Tortuguero & Onomatopéyico \\
\hline Porphyrio martinica & Polla de agua & Las Juntas Abangares & Comportamiento \\
\hline \multicolumn{4}{|l|}{ Aramidae } \\
\hline \multirow[t]{2}{*}{ Aramus guarauna } & Carao & Guanacaste & Onomatopéyico \\
\hline & Correa & Guanacaste & Onomatopéyico \\
\hline \multicolumn{4}{|l|}{ Burhinidae } \\
\hline Burhinus bistriatus & Alcaravan & Guanacaste & \\
\hline \multicolumn{4}{|l|}{ Recurvirostridae } \\
\hline Himantopus mexicanus & Soldadito & Puntarenas & Físico \\
\hline \multicolumn{4}{|l|}{ Jacanidae } \\
\hline Jacana spinosa & Yegüita & Guanacaste & Físico \\
\hline \multicolumn{4}{|l|}{ Scolopacidae } \\
\hline Actitis macularia & Alza colitas & Costa Rica & Comportamiento \\
\hline \multicolumn{4}{|l|}{ Columbidae } \\
\hline Columba livia & Paloma de Castilla & Pajareros & Geográfico \\
\hline Patagioenas cayennensis & Paloma morada & Pajareros & Físico \\
\hline Patagioenas speciosa & Paloma morada & San Vito & Físico \\
\hline Patagioenas flavirostris & Paloma morada & Pajareros & Físico \\
\hline Patagioenas fasciata & Paloma collareja & Pajareros & Físico \\
\hline Patagioenas subvinacea & Paloma morada & Pajareros & Físico \\
\hline \multirow[t]{2}{*}{ Patagioenas nigrirostris } & Dos tontos son & Tortuguero & Onomatopéyico \\
\hline & Paloma morada & Pajareros & Físico \\
\hline \multirow[t]{2}{*}{ Zenaida asiatica } & Ala blanca & Valle Central & Físico \\
\hline & Arrocera & Valle Central & Comportamiento \\
\hline \multirow[t]{2}{*}{ Columbina inca } & San Juan & Valle Central & Onomatopéyico \\
\hline & Tortola & Valle Central & Físico \\
\hline
\end{tabular}




\begin{tabular}{|c|c|c|c|}
\hline Columbina passerina & Tortolita & Valle Central & Físico \\
\hline Columbina talpacoti & Tortolita & Guanacaste & Físico \\
\hline \multirow[t]{2}{*}{ Leptotila verreauxi } & Cola Blanca & Tausito & Físico \\
\hline & Yuré & Valle Central & Onomatopéyico \\
\hline \multicolumn{4}{|l|}{ Psittacidae } \\
\hline \multirow[t]{3}{*}{ Aratinga finschi } & Chucuyo & Valle Central & \\
\hline & Perico cola larga & Golfito & Físico \\
\hline & Perico de palmera & Las Juntas Abangares & Comportamiento \\
\hline Aratinga canicularis & Catano & Las Juntas Abangares & \\
\hline Ara ambiguus & Lapa Verde & Costa Rica & Físico \\
\hline Ara macao & Lapa Roja & Costa Rica & Físico \\
\hline \multirow[t]{2}{*}{ Brotogeris jugularis } & Chimbolito & Golfito & Onomatopéyico \\
\hline & Zapollol & Las Juntas Abangares & \\
\hline \multirow[t]{2}{*}{ Pionus senilis } & Cotorra & Valle Central & Físico \\
\hline & Chucuyo & Valle Central & \\
\hline Amazona albifrons & Kankan & Las Juntas Abangares & Onomatopéyico \\
\hline Amazona autumnalis & Lora jupa roja & Pajareros & Físico \\
\hline \multirow{2}{*}{ Amazona farinosa } & Lora cabeza negra & San Vito & Físico \\
\hline & Lora jupa gris & Pajareros & Físico \\
\hline Amazona auropalliata & Lora jupa amarilla & Pajareros & Físico \\
\hline \multicolumn{4}{|l|}{ Cuculidae } \\
\hline \multirow[t]{3}{*}{ Piaya cayana } & Boo chizo & Valle Central & Comportamiento \\
\hline & Cacao & San Vito & Físico \\
\hline & San Miguel & Las Juntas Abangares & Onomatopéyico \\
\hline \multirow[t]{3}{*}{ Tapera naevia } & Rondero & San Vito & Comportamiento \\
\hline & Tres pesos pido & Puriscal & Onomatopéyico \\
\hline & Ave de la Animas & Buenos Aires & Comportamiento \\
\hline Morococcyx erythropygius & Horero & Las Juntas Abangares & Comportamiento \\
\hline Crotophaga ani & Tijo & Zona sur & Onomatopéyico \\
\hline \multirow[t]{3}{*}{ Crotophaga sulcirostris } & Tijo & Valle Central & Onomatopéyico \\
\hline & Tinko & Guanacaste & Onomatopéyico \\
\hline & Zopilotillo & Valle Central & Físico \\
\hline \multicolumn{4}{|l|}{ Tytonidae } \\
\hline \multirow[t]{2}{*}{ Tyto alba } & Lechuza de campanario & Valle Central & Comportamiento \\
\hline & Lechuza ratonera & Valle Central & Comportamiento \\
\hline \multicolumn{4}{|l|}{ Strigidae } \\
\hline Otus choliba & Estucurucu & Valle Central & Onomatopéyico \\
\hline Otus clarkii & Sorococa & Las Juntas Abangares & Onomatopéyico \\
\hline Pulsatrix perspicillata & Oropopo & Las Juntas Abangares & Onomatopéyico \\
\hline \multirow[t]{2}{*}{ Glaucidium brasilianum } & Cuatro ojos & Valle Central & Físico \\
\hline & Majafierro & Valle Central & \\
\hline \multicolumn{4}{|l|}{ Caprimulgidae } \\
\hline Nyctidromus albicollis & Cuyeo & Costa Rica & Onomatopéyico \\
\hline
\end{tabular}




\begin{tabular}{|c|c|c|c|}
\hline Caprimulgus saturatus & Tres cobijas & Cerro de la Muerte & Onomatopéyico \\
\hline \multicolumn{4}{|l|}{ Nyctibiidae } \\
\hline Nyctibius grandis & Pájaro estaca & Tortuguero & Físico \\
\hline Nyctibius griseus & Pájaro estaca & San Vito & Físico \\
\hline Nyctibius jamaicensis & Gozona & Guanacaste & Onomatopéyico \\
\hline \multicolumn{4}{|l|}{ Trochilidae } \\
\hline Amazilia tzacatl & Gorrión & Valle Central & \\
\hline \multicolumn{4}{|l|}{ Trogonidae } \\
\hline Trogon massena & Caicota & Osa, Pto Jiménez & Onomatopéyico \\
\hline \multirow[t]{2}{*}{ Pharomachrus mocinno } & Quetzal & Costa Rica & \\
\hline & Coludo & Cerro de la Muerte & Físico \\
\hline \multicolumn{4}{|l|}{ Momotidae } \\
\hline \multirow{2}{*}{ Momotus momota } & Boo & Costa Rica & Comportamiento \\
\hline & Boo azul & Costa Rica & Físico \\
\hline Eumomota superciliosa & Boo & Guanacaste & Comportamiento \\
\hline \multicolumn{4}{|l|}{ Ramphastidae } \\
\hline Eubucco bourcierii & Ruano & Río cuarto & Físico \\
\hline Semnornis frantzii & Cocora & Valle Central & Onomatopéyico \\
\hline Aulacorhynchus prasinus & Curre & Valle Central & Onomatopéyico \\
\hline \multirow[t]{2}{*}{ Pteroglossus torquatus } & Curre & Tortuguero & Onomatopéyico \\
\hline & Tucán Feliz & La Cruz Guanacaste & Onomatopéyico \\
\hline \multirow[t]{2}{*}{ Pteroglossus frantzii } & Cachis & San Vito & Onomatopéyico \\
\hline & Cusingo & Pérez Zeledón & \\
\hline \multirow[t]{2}{*}{ Ramphastos sulfuratus } & Pico arcoiris & Pajareros & Físico \\
\hline & Pico iris & Tortuguero & Físico \\
\hline \multirow[t]{2}{*}{ Ramphastos swainsonii } & Dios te de & Atlántico & Onomatopéyico \\
\hline & Quioro & Atlántico & Onomatopéyico \\
\hline \multicolumn{4}{|l|}{ Picidae } \\
\hline Picumus olivaceus & Telegrafista & Zona sur & Onomatopéyico \\
\hline Melanerps formicivorus & Payasito & Cerro de la Muerte & Físico \\
\hline Campephilus guatemalensis & Dos golpes & Guanacaste & Onomatopéyico \\
\hline \multicolumn{4}{|l|}{ Furnariidae } \\
\hline Margarornis rubiginosus & Chupa musgo & Dota & Comportamiento \\
\hline \multicolumn{4}{|l|}{ Tyrannidae } \\
\hline Elaenia flavogaster & Tontillo & Valle Central & Físico \\
\hline \multirow[t]{2}{*}{ Todirostrum cinereum } & Espatulilla & Las Juntas Abangares & Comportamiento \\
\hline & Mantequillilla & Guápiles & Comportamiento \\
\hline Contopus cooperii & Tontillo & Valle Central & Físico \\
\hline Contopus sordidulus & Tontillo & Valle Central & Físico \\
\hline Contopus virens & Tontillo & Valle Central & Físico \\
\hline Contopus cinereus & Tontillo & Valle Central & Físico \\
\hline Myiarchus tuberculifer & Tontillo & Valle Central & Físico \\
\hline Myiarchus crinitus & Tontillo & Valle Central & Físico \\
\hline Pitangus sulphuratus & Cristo Fue & Costa Rica & Onomatopéyico \\
\hline
\end{tabular}




\begin{tabular}{|c|c|c|c|}
\hline & Pecho amarillo & Costa Rica & Físico \\
\hline Megarhynchus pitangua & Pecho amarillo & Costa Rica & Físico \\
\hline Myiozetetes similis & Pecho amarillo & Costa Rica & Físico \\
\hline Myiozetetes granadensis & Pecho amarillo & Costa Rica & Físico \\
\hline Myiodynastes luteiventris & Pecho amarillo & Costa Rica & Físico \\
\hline Conopias albovittata & Pecho amarillo & Costa Rica & Físico \\
\hline Tyrannus melancholicus & Pecho amarillo & Costa Rica & Físico \\
\hline Tyrannus verticalis & Pecho amarillo & Costa Rica & Físico \\
\hline Tyrannus tyrannus & Viuda & Heredia & Físico \\
\hline Tyrannus forficatus & Tijerillas & Zona Sur & Físico \\
\hline Tyrannus savana & Tijerillas & Las Juntas Abangares & Físico \\
\hline \multicolumn{4}{|l|}{ Incertae sedis } \\
\hline \multirow[t]{2}{*}{ Tityra semifasciata } & Calandria & San Vito & \\
\hline & Pájaro chancho & Valle Central & Onomatopéyico \\
\hline \multicolumn{4}{|l|}{ Cotingidae } \\
\hline Cephalopterus glabricollis & Pájaro sombrilla & Monteverde & Físico \\
\hline \multirow[t]{2}{*}{ Procnias tricarunculata } & Calandria & Pajareros & \\
\hline & Pájaro Campana & Monteverde & Onomatopéyico \\
\hline \multicolumn{4}{|l|}{ Pipridae } \\
\hline \multirow{2}{*}{ Manacus candei } & Quiebra palos & Pajareros & Onomatopéyico \\
\hline & Quiebra ramas & Tortuguero & Onomatopéyico \\
\hline Manacus aurantiacus & Quiebra palos & Pajareros & Onomatopéyico \\
\hline Chiroxiphia linearis & Toledo & Costa Rica & Onomatopéyico \\
\hline \multicolumn{4}{|l|}{ Vireonidae } \\
\hline \multirow[t]{2}{*}{ Vireo flavoviridis } & Chiguisa & Barva & Onomatopéyico \\
\hline & Chuesa & Puriscal & Onomatopéyico \\
\hline Vireo olivaceus & Chiguisa & Barva & Onomatopéyico \\
\hline \multicolumn{4}{|l|}{ Corvidae } \\
\hline Calocitta formosa & Urraca & Guanacaste & Físico \\
\hline Cyanocorax morio & Piapia & Valle Central & Onomatopéyico \\
\hline \multicolumn{4}{|l|}{ Hirundinidae } \\
\hline Pigochelidon cyanoleuca & Golondrina & Costa Rica & \\
\hline \multicolumn{4}{|l|}{ Troglodytidae } \\
\hline \multirow[t]{2}{*}{ Campylorhynchus rufinucha } & Chico piojo & Guanacaste & Onomatopéyico \\
\hline & Botijon & Mora & \\
\hline \multirow[t]{2}{*}{ Thryothorus modestus } & Chinchirigui & Costa Rica & Onomatopéyico \\
\hline & Huevos blancos & San Ramón de la Virgen & Físico \\
\hline \multirow[t]{2}{*}{ Troglodytes aedon } & Cucarachero & & Comportamiento \\
\hline & Soterre & Costa Rica & \\
\hline \multicolumn{4}{|l|}{ Silviidae } \\
\hline Polioptila albiloris & Espatulilla & Las Juntas Abangares & Comportamiento \\
\hline Polioptila plumbea & Espatulilla & Las Juntas Abangares & Comportamiento \\
\hline \multicolumn{4}{|l|}{ Turdidae } \\
\hline Myadestes melanops & Jilguero & Pajareros & Onomatopéyico \\
\hline Catharus gracilirostris & Cuitiento & Cerro de la Muerte & Comportamiento \\
\hline
\end{tabular}




\begin{tabular}{|c|c|c|c|}
\hline Catharus aurantiirostris & Jilguerillo de charral & Valle Central & Geográfico \\
\hline Catharus fuscater & Arremedon & Pajareros & Onomatopéyico \\
\hline \multirow[t]{2}{*}{ Catharus frantzii } & Jilguerillo de montaña & Pajareros & Geográfico \\
\hline & Jilguerillo de ronda & Coronado & Geográfico \\
\hline Catharus fuscescens & Conchita & $\begin{array}{l}\text { San Rafael Heredia, } \\
\text { Pajareros }\end{array}$ & Físico \\
\hline Catharus minimus & Conchita & $\begin{array}{l}\text { San Rafael Heredia, } \\
\text { Pajareros }\end{array}$ & Físico \\
\hline Catharus ustulatus & Conchita & $\begin{array}{l}\text { San Rafael Heredia, } \\
\text { Pajareros }\end{array}$ & Físico \\
\hline Turdus nigrescens & Escarchero & Poás, Cerro de la Muerte & Físico \\
\hline Turdus plebejus & Yigüirro de montaña & & Geográfico \\
\hline Turdus obsoletus & Yigüirro & Guápiles & \\
\hline Turdus grayi & Yigüirro & Costa Rica & \\
\hline \multirow[t]{2}{*}{ Turdus assimilis } & Yigüirro collarejo & San Vito & Físico \\
\hline & Yigüirro de montaña & Pajareros & Geográfico \\
\hline \multicolumn{4}{|l|}{ Bombycillidae } \\
\hline Bombycilla cedrorum & Jilguero chino & Heredia & Físico \\
\hline \multicolumn{4}{|l|}{ Ptilogonatidae } \\
\hline Phainoptila melanoxantha & Come mora & Cerro de la Muerte & Comportamiento \\
\hline \multirow[t]{3}{*}{ Ptilogonys caudatus } & Pitorreal & Heredia & Onomatopéyico \\
\hline & Timbre & Cerro de la Muerte & Onomatopéyico \\
\hline & Coronel & Cerro de la Muerte & Físico \\
\hline \multicolumn{4}{|l|}{ Parulidae } \\
\hline Vermivora peregrina & Cazadorcita & Heredia & Comportamiento \\
\hline Dendroica petechia & Canarita & Uruca & Físico \\
\hline Mniotilta varia & Cebrita & Heredia & Físico \\
\hline Wilsonia pusilla & Curruquitas & Cerro de la Muerte & Físico \\
\hline Myioborus miniatus & Candelita & Heredia & Físico \\
\hline Myioborus torquatus & Amigo de hombre & Cerro de la Muerte & Comportamiento \\
\hline \multicolumn{4}{|l|}{ Thraupidae } \\
\hline Chlorospingus ophtalmicus & Cuatro ojos & Carrizal & Físico \\
\hline Habia rubica & Relicario & San Rafael Heredia & \\
\hline Piranga flava & Cardenal & Pajareros & Físico \\
\hline \multirow[t]{3}{*}{ Piranga rubra } & Cardenal & Valle Central & Físico \\
\hline & Pan de achiote & San Ramón de la Virgen & Físico \\
\hline & Sangre toro & Guanacaste & Físico \\
\hline \multirow[t]{2}{*}{ Piranga olivacea } & Cardenal & Pajareros & Físico \\
\hline & Cardenal alas negras & Valle Central & Físico \\
\hline Piranga bidentata & Cardenal alas negras & Valle Central & Físico \\
\hline Ramphocelus sanguinolentus & Rey de Sargento & Pajareros & Físico \\
\hline Ramphocelus passerinii & Sargento & Pajareros & Físico \\
\hline Ramphocelus costaricensis & Sargento & Pajareros & Físico \\
\hline Thraupis episcopus & Viudita & Costa Rica & \\
\hline
\end{tabular}




\begin{tabular}{|c|c|c|c|}
\hline Thraupis palmarum & Viuda de la costa & Pajareros & Geográfico \\
\hline Bangsia arcaei & Aguión & Pajareros & Físico \\
\hline \multirow[t]{2}{*}{ Tangara icterocephala } & Chia & Purisil & Onomatopéyico \\
\hline & Rayo de sol & Pajareros & Físico \\
\hline Tangara guttata & Cebra & Pajareros & Físico \\
\hline Tangara gyrola & Pavito & Pajareros & Físico \\
\hline \multirow[t]{3}{*}{ Tangara larvata } & Juana & San Ramón de la Virgen & Onomatopéyico \\
\hline & Mariposa & Pajareros & Físico \\
\hline & Siete colores & Pajareros & Físico \\
\hline Tangara dowii & Mariposa de clima frió & Pajareros & Geográfico \\
\hline Dacnis venusta & Calzones rojos & Pajareros & Físico \\
\hline Dacnis cayana & Rey de Viuda & Pajareros & Físico \\
\hline \multirow[t]{2}{*}{ Chlorophanes spiza } & Rey de mar & Pajareros & Físico \\
\hline & Verde mar & San Ramón de la Virgen & Físico \\
\hline Cyanerpes lucidus & Picudo pata amarilla & Pajareros & Físico \\
\hline \multirow[t]{3}{*}{ Cyanerpes cyaneus } & Mielero & Limón & Comportamiento \\
\hline & Picudo pata roja & Pajareros & Físico \\
\hline & Tucuso & Pérez Zeledón & \\
\hline \multicolumn{4}{|l|}{ Emberizidae } \\
\hline \multirow[t]{2}{*}{ Volatinia jacarina } & Brea & Guanacaste, Valle Central & Físico \\
\hline & Piusillo negro & San Ramón de la Virgen & Físico \\
\hline \multirow[t]{4}{*}{ Sporophila americana } & Arrocerito & Puerto Viejo Sarapiquí & Comportamiento \\
\hline & Monjito & Golfito & Físico \\
\hline & Zetillero collarejo & Guanacaste & Físico \\
\hline & Zetillero & Guanacaste & Comportamiento \\
\hline Sporophila torqueola & Zetillero collarejo & Limón & Físico \\
\hline Sporophila nigricollis & Zetillero panameño & Pajareros & Geográfico \\
\hline Sporophila minuta & Zetillero ladrillo & Pajareros & Físico \\
\hline Tiaris olivacea & Gallito & Pajareros & Comportamiento \\
\hline Pselliophorus tibialis & Calzones amarillos & Pajareros & Físico \\
\hline Arremon aurantirrostris & Pico chile & Kekoldi & Físico \\
\hline Arremonops rufivirgatus & Timbón & Las Juntas Abangares & Físico \\
\hline \multirow[t]{2}{*}{ Arremonops conirostris } & Pájaro susuki & Puerto Viejo Limón & Onomatopéyico \\
\hline & Purisquero & Puriscal & Físico \\
\hline \multirow[t]{2}{*}{ Melozone biarcuatum } & Cuatro ojos jupa roja & Heredia & Físico \\
\hline & Rey de come maíz & Carrizal & Físico \\
\hline \multirow[t]{2}{*}{ Melozone leucotis } & Cuatro ojos & Heredia & Físico \\
\hline & Payasito & Valle Central & Físico \\
\hline Aimophila ruficauda & Pájaro chicle & Parrita & \\
\hline \multirow[t]{2}{*}{ Zonotrichia capensis } & Comemaíz & Valle Central & Comportamiento \\
\hline & Pirris & Cerro de la Muerte & Onomatopéyico \\
\hline \multicolumn{4}{|l|}{ Cardenalidae } \\
\hline \multirow[t]{2}{*}{ Saltator coerulescens } & Come puntas & Heredia & Comportamiento \\
\hline & Come chayote & Carrizal & Comportamiento \\
\hline
\end{tabular}




\begin{tabular}{|c|c|c|c|}
\hline \multirow[t]{3}{*}{ Saltator maximus } & Chayotero & Guápiles & Comportamiento \\
\hline & Come puntas & Heredia & Comportamiento \\
\hline & Chojui & Guápiles & Onomatopéyico \\
\hline Saltator atriceps & Chayotero & Guápiles & Comportamiento \\
\hline \multirow[t]{2}{*}{ Pheucticus tibialis } & Chorcho & Perez Zeledón & Onomatopéyico \\
\hline & Maizero & Pajareros & Comportamiento \\
\hline Pheucticus ludovicianus & Calandrio & Pajareros & \\
\hline Caryothraustes poliogaster & Sarapiqueña & Alajuela & Geográfico \\
\hline Passerina caerulea & Alondra & Pajareros & \\
\hline Passerina cyanea & Inrris & Pajareros & \\
\hline Passerina ciris & Arcoiris & Pajareros & Físico \\
\hline Spiza americana & Arrocero & Las Juntas Abangares & Comportamiento \\
\hline \multicolumn{4}{|l|}{ Icteridae } \\
\hline & Sargento & San Carlos & Físico \\
\hline & Zacatera & Valle Central & Comportamiento \\
\hline & Zanate & Costa Rica & \\
\hline & Pius & Valle Central & Onomatopéyico \\
\hline & Chorcha del platanar & Sarapiquí & Físico \\
\hline & Cacique ahumado & Guanacaste, Valle Central & Físico \\
\hline & Chorcha & Pajareros & \\
\hline & Chilchote & Las Juntas Abangares & \\
\hline & Chilto & Las Juntas Abangares & \\
\hline & Chorchin & Las Juntas Abangares & \\
\hline & Balsero & Las Juntas Abangares & Comportamiento \\
\hline & Cacique & Valle Central & \\
\hline & Naranjero & Guápiles & Comportamiento \\
\hline & Pico de plata & Valle Central & Físico \\
\hline Cacique uropygialis & Sargento & & Físico \\
\hline \multirow[t]{3}{*}{ Psarocolius wagleri } & Oropéndola & Limón & \\
\hline & Oropel & Orosi & Físico \\
\hline & Guacalillo & Tausito & Onomatopéyico \\
\hline Psarocolius montezuma & Oropéndola & Valle Central & \\
\hline \multicolumn{4}{|l|}{ Fringillidae } \\
\hline Carduelis xanthogastra & Mozotillo de montaña & Pajareros & Geográfico \\
\hline Carduelis psaltria & Mozotillo de charral & Guanacaste, pajareros & Geográfico \\
\hline Euphonia affinis & Finito & Las Juntas Abangares & Onomatopéyico \\
\hline Euphonia luteicapilla & Monjito & Pajareros & Físico \\
\hline Euphonia laniirostris & Agüio & Pajareros & \\
\hline Euphonia hirundinacea & Agüio & Pajareros & \\
\hline \multirow[t]{2}{*}{ Euphonia elegantissima } & Cacique veranero & Patarra, Cartago & Físico \\
\hline & Veranero & Heredia & Físico \\
\hline Euphonia gouldi & Barranquillo (a) & Pajareros & \\
\hline
\end{tabular}




\begin{tabular}{llll}
\hline & Culo rojo & San Ramón de la Virgen & Físico \\
& Güere & Turrialba & Físico \\
Euphonia minuta & Canarito & Pajareros & Físico \\
& Finito canario & Pajareros & Físico \\
Chlorophonia callophrys & Rey de Rualdo & Pajareros & Físico \\
& Rualdo & Pajareros & \\
\hline Passeridae & & & \\
\hline Passer domesticus & Electricista & Valle Central & Comportamiento \\
\hline
\end{tabular}

\section{Conclusión}

La presente investigación demostró la gran diversidad de nombres con que se bautiza a las aves del país y cómo se utilizan diferentes características para nombrar a la misma especie en diferentes lugares. Al ser una compilación amplia de nombres comunes, sin ser definitiva, permite observar diferentes sinónimos para cada especie, lo que facilitará la comunicación entre ornitólogos y las demás personas. En los procesos actuales de conservación, donde se trata de involucrar de forma más activa a las comunidades, el conocer y poseer nombres comunes para las especies tanto animales como plantas, permitirá una integración más fácil. Es por eso que este tipo de trabajos es de vital importancia para dichos fines.

\section{Bibliografía}

Banks, R., C. et al. 2002. "Forty-third supplement to the American Ornithologist' Union checklist of North American Birds". Auk. 119: 897-906.

Fournier, Luis y Elmer García. 1998. Nombres vernaculares y científicos de Costa Rica. San José: Editorial Guayacán.

Härlin, M. 2003. "Taxon names as paradigms: the structure of nomenclatural revolutions". Cladistics. 19: 138-143.

Mones, A. y P. Kuhl de Mones. 1991. "Comentarios sobre el uso de nombres vulgares en zoología”. Anales del Instituto Biológico de la Universidad Autónoma de México, Series Zoológicas. 62: 249-252.

Mora, José. 2000. Mamíferos silvestres de Costa Rica. San José: EUNED.

Polaszek, A y E. O. Wilson. 2005. "Sense and stability in animal names". TRENDS in Ecology and Evolution. 20: 421-422. 
Quesada-Monge, R y J. Fernández-Vega. 2005. “Actualización de listado de especies arbóreas de uso forestal y otros usos en Costa Rica”. Kurú. 2: 1-45.

Savage, James. 2002. The amphibians and reptiles of Costa Rica: a herpetofauna between two continents, between two seas. Chicago: University of Chicago Press.

Stiles, Gary y Alexander Skutch. 1989. Guide to the birds of Costa Rica. New York: Cornell University Press.

Vuilleumier, F. 1999. "Sobre la necesidad de estandarizar los nombres en castellano y portugués de las aves neotripicales". Ornitología Neotropical. 10: 69-75. 
